\title{
Broader, flatter optical spectra of passively mode-locked semiconductor lasers for a wavelength-division multiplexing source
}

\author{
Danny Eliyahu, Amnon Yariv, and Randal A. Salvatore
}

\begin{abstract}
Using the time domain master equation for a complex electric-field pulse envelope, we find analytical results for the optical spectra of passively mode-locked semiconductor lasers. The analysis includes the effect of optical nonlinearity of semiconductor lasers, which is characterized by a slow saturable amplifier and absorber. Group velocity dispersion, bandwidth limiting, and self-phase modulation were considered as well. The FWHM of the spectrum profile was found to have a strong dependence on group velocity dispersion and self-phase modulation. For large absolute values of the chirp parameter, the optical spectra result in equispaced continuous wave frequencies, a large fraction of which have equal power. (C) 1997 Optical Society of America
\end{abstract}

Wavelength-division multiplexing (WDM) has become the technology of choice to fully utilize the enormous bandwidth $(\sim 12 \mathrm{THz})$ of single-mode fibers for communication purposes. WDM systems require light sources with precisely determined optical wavelengths. Such a system contains complicated control circuits together with many lasers. Alternatively, there has been much recent interest in the use of mode-locked lasers as optical sources for WDM networks ${ }^{1,2}$; in particular, the use of $\operatorname{InGaAsP} / \mathrm{InP}$ semiconductor mode-locked lasers operating at wavelengths ${ }^{3,4}$ around $1.55 \mu \mathrm{m}$, the low-loss wavelength of single-mode fiber.

A mode-locked laser emits a field that consists of a large number of equally spaced, continuous-wave frequencies that can be separated and modulated and act as individual monochromatic sources of a WDM source. In this paper we study the steady-state characteristics of the optical spectrum profile of long monolithic ${ }^{5}$ (and external cavity) passively modelocked semiconductor lasers, as well as the possibility of utilizing them as WDM sources. We assume that

D. Eliyahu and A. Yariv are with the California Institute of Technology, M/S 128-95, Pasadena, California 91125. R. A. Salvatore is with the Department of Electrical and Computer Engineering, University of California, Santa Barbara, California 93106.

Received 15 September 1996; revised manuscript received 13 November 1996.

0003-6935/97/153430-05\$10.00/0

(C) 1997 Optical Society of America a large number of lasing longitudinal modes are present and short pulses are produced. These lasers include at least two sections: the long laser section is forward biased to provide the gain, and by reversebiasing the short segment of the laser, one can form a waveguide saturable absorber. Mode-locked semiconductor lasers are characterized by relaxation times of gain and absorber nonlinearity that are long compared to pulse duration, ${ }^{5}$ i.e., the slow saturable effects are energy dependent. We start with the time domain differential equation. ${ }^{6-8}$ The master equation, given by ${ }^{8}$

$$
\begin{aligned}
& \left(l _ { t } \left[1+\left(\frac{2 \Delta \omega}{\Omega}\right)^{2}+i \frac{2 \Delta \omega}{\Omega}+2\left(1-4 i \frac{\Delta \omega}{\Omega}\right) \frac{1}{\Omega}\left(\frac{\mathrm{d}}{\mathrm{d} t}\right)\right.\right. \\
& \left.-\frac{4}{\Omega^{2}}\left(\frac{\mathrm{d}^{2}}{\mathrm{~d} t^{2}}\right)\right]+\left\{\left(1+i \alpha_{l}\right) l \exp \left[-\frac{E(t)}{E_{\mathrm{sl}}}\right]\right\}-\left\{\left(1+i \alpha_{g}\right) g\right. \\
& \left.\left.\quad \times \exp \left[-\frac{E(t)}{E_{\mathrm{sg}}}\right]\right\}+\left[i\left(\varphi-D \frac{\mathrm{d}^{2}}{\mathrm{~d} t^{2}}\right)\right]+\Delta T \frac{\mathrm{d}}{\mathrm{d} t}\right) a(t)=0,
\end{aligned}
$$

describes the action of a bandwidth-limited linearcavity loss, a slow saturable absorber, a slow saturable gain, and dispersion on the complex pulse electricfield envelope [the first set of brackets, the first set of braces, the second set of braces, and the fourth set of brackets in Eq. (1), respectively]. Self-phase modulation (SPM) of the gain and absorption sections is introduced by the amplitude-to-phase coupling parameters $\alpha_{g}$ and $\alpha_{l}$, respectively. $\Delta T$ represents the time delay of the pulse with respect to a passive 
cavity delay and the delay of linear dispersion (group velocity). It is related to repetition rate detuning. $D=\Sigma_{m} \beta_{m}{ }^{\prime \prime} L_{m}$, the effective group-velocity dispersion (GVD) of a round trip in the Fabry-Perot cavity, is given by the sum over GVD of individual elements, where $\beta_{m}$ " is the GVD per unit length of the $m$ th element, $L_{m}$ is its length, $l_{t}$ is the linear (field) cavity loss of the system, $\varphi$ is a constant phase shift, $\Delta \omega$ is the change in the carrier angular frequency from that of peak net gain (detuning of the central angular frequency), $\Omega$ is the gain bandwidth, and $g$ refers to the saturated gain preceding the pulse. The saturable gain is related to the small-signal (unsaturated) gain $g_{0}$ by

$$
g=g_{0}\left[\frac{\exp \left(T / \tau_{g}\right)-1}{\exp \left(T / \tau_{g}\right)-\exp \left(-E / E_{\mathrm{sg}}\right)}\right],
$$

where $T$ is the time between adjacent pulse arrivals in the saturable amplifier. After passage of the pulse, the gain relaxes with relaxation time $\tau_{g}$. In Eq. (2), $E_{\mathrm{sg}}$ is the saturation energy of the gain whereas $E=E(\infty)$ is the total energy of the pulse, where the pulse energy that has passed through the gain at time $t$ is

$$
E(t)=\int_{-\infty}^{t}\left|a\left(t^{\prime}\right)\right|^{2} \mathrm{~d} t^{\prime}
$$

$a(t)$ denotes the pulse electric-field envelope and is normalized such that $|a(t)|^{2}$ describes the power of the optical field. Similarly, as in Eq. (2), the saturated absorption preceding the pulse $l$ is related to the small-signal absorption $l_{0}$ through the relaxation time $\tau_{l}\left(<\tau_{g}\right)$ and the energy saturation $E_{\mathrm{sl}}$ of the absorber.

In general the stability parameter ${ }^{9} s=E_{\mathrm{sg}} / E_{\mathrm{sl}}$ is much larger than 1 , and in order to achieve analytical results, we study the case of low energy, i.e., $E<E_{\text {sl }}$. In this case the exponents in Eq. (1) are expanded to first order in a Taylor series for gain and to second order for absorption. Therefore Eq. (1) takes the form

$$
\left\{l_{t}+4 l_{t}\left(\frac{\Delta \omega}{\Omega}\right)^{2}+\left(1+i \alpha_{l}\right) l-\left(1+i \alpha_{g}\right) g+i\left(2 l_{t} \frac{\Delta \omega}{\Omega}+\varphi\right)\right.
$$

$$
\begin{gathered}
+\left[-\left(1+i \alpha_{l}\right) l+\left(1+i \alpha_{g}\right) \frac{g}{s}\right] \frac{E(t)}{E_{\mathrm{sl}}}+\frac{1}{2}\left(1+i \alpha_{l}\right) \\
\quad \times l\left[\frac{E(t)}{E_{\mathrm{sl}}}\right]^{2}+2\left(l_{t}+\frac{\Omega \Delta T}{2}-4 i l_{t} \frac{\Delta \omega}{\Omega}\right) \frac{1}{\Omega}\left(\frac{\mathrm{d}}{\mathrm{d} t}\right) \\
\left.-\left(4 l_{t}+i \Omega^{2} D\right) \frac{1}{\Omega^{2}}\left(\frac{\mathrm{d}^{2}}{\mathrm{~d} t^{2}}\right)\right\} a(t)=0
\end{gathered}
$$

We restrict the solution to that of a stable pulse train. In this case we assume

$$
a(t)=A[\operatorname{sech}(t / \tau)]^{1+i \beta},
$$

where $\tau$ is related to the pulse width, $A=(E / 2 \tau)^{1 / 2}$ is the electric-field amplitude, and $\beta$ is the chirp parameter.

Substituting Eq. (5) into Eq. (4) and solving for the unknown parameters, we get

$$
\begin{aligned}
\beta= & \frac{1}{2\left(\Omega^{2} D-4 l_{t} \alpha_{l}\right)}\left\{3\left(4 l_{t}+\Omega^{2} D \alpha_{l}\right)-\left[9\left(4 l_{t}+\Omega^{2} D \alpha_{l}\right)^{2}\right.\right. \\
& \left.\left.+8\left(\Omega^{2} D-4 l_{t} \alpha_{l}\right)^{2}\right]^{1 / 2}\right\} \\
\frac{E}{E_{\mathrm{sl}}}= & \frac{1}{2 \gamma_{1}}\left[-\gamma_{2}+\left(\gamma_{2}^{2}-4 \gamma_{1} \gamma_{3}\right)^{1 / 2}\right] \\
\Omega \tau= & 4\left(\frac{4 l_{t}-2 \beta^{2} l_{t}-3 \beta D / 2 \Omega^{2}}{l}\right)^{1 / 2}\left(\frac{E_{\mathrm{sl}}}{E}\right) \\
\frac{\Delta \omega}{\Omega}= & -\frac{\Omega \tau}{8 l_{t}\left(1+2 \beta^{2}\right)}\left\{l\left(\beta-\alpha_{l}\right)\left[1+\frac{l}{2}\left(\beta-\alpha_{l}\right)\left(\frac{E}{E_{\mathrm{sl}}}\right)^{2}\right]\right. \\
& \left.-\frac{g}{s}\left(\beta-\alpha_{g}\right) \frac{E}{E_{\mathrm{sl}}}\right\}, \\
\Omega \Delta T= & -2 l_{t}-8 l_{t} \beta \frac{\Delta \omega}{\Omega}-\frac{1}{2} \Omega \tau\left(l-\frac{g}{s}\right) \frac{E}{E_{\mathrm{sl}}} \\
& +\frac{1}{4} l \Omega \tau\left(\frac{E}{E_{\mathrm{sl}}}\right)^{2}, \\
\varphi= & -2 l_{t} \frac{\Delta \omega}{\Omega}+\frac{8 l_{t} \beta}{(\Omega \tau)^{2}}+\frac{D\left(1-\beta^{2}\right)}{\tau^{2}}-l \alpha_{l}+g \alpha_{g} \\
& +\frac{1}{2}\left(l \alpha_{l}-\frac{g \alpha_{g}}{s}\right) \frac{E}{E_{\mathrm{sl}}}-\frac{1}{4} l \alpha_{l}\left(\frac{E}{E_{\mathrm{sl}}}\right)^{2}
\end{aligned}
$$

where

$$
\begin{aligned}
& \gamma_{1}=\frac{l}{8 l_{t}}\left[\frac{3-\beta D \Omega^{2} / l_{t}-\beta^{2}}{2-\beta^{2}-3 \beta D \Omega^{2} / 4 l_{t}}+\frac{\left(\beta-\alpha_{l}\right)^{2}\left(2-\beta^{2}-3 \beta D \Omega^{2} / 4 l_{t}\right)}{\left(1+2 \beta^{2}\right)^{2}}\right], \\
& \gamma_{2}=-\frac{1}{2 l_{t}}\left\{l-\frac{g}{s}+\frac{\left[-l \alpha_{l}+g \alpha_{g} / s+\beta(l-g / s)\right]\left(\beta-\alpha_{l}\right)\left(2-\beta^{2}-3 \beta D \Omega^{2} / 4 l_{t}\right)}{\left(1+2 \beta^{2}\right)^{2}}\right\}, \\
& \gamma_{3}=1+\frac{l-g}{l_{t}}+\frac{1}{2 l l_{t}\left(1+2 \beta^{2}\right)^{2}}\left[\left(\beta-\alpha_{l}\right) l-\left(\beta-\alpha_{g}\right) \frac{g}{s}\right]^{2}\left(2-\beta^{2}-3 \beta D \Omega^{2} / 4 l_{t}\right) .
\end{aligned}
$$


Note that, although this analytic theory [Eqs. (4)(11)] assumes that $E<E_{\mathrm{sl}}$, the result it predicts is reasonable for ${ }^{6,10} E \simeq E_{\mathrm{sl}}$ and higher, depending on the laser's parameters. The contribution of $\alpha_{g}$ to the chirp parameter is negligible since its relative contribution is smaller by a factor of $s^{2}$ than that of $\alpha_{l}$. Transform-limited pulses $(\beta=0)$ are found for $D=$ $D_{0} \equiv 4 l_{t} \alpha_{l} / \Omega^{2}$, whereas a small up-chirp $\left(\beta \simeq 3 \alpha_{l} / 2-\right.$ $\left.\left(9 \alpha_{l}^{2} / 4+2\right)^{1 / 2}<0\right)$ is achieved for $D \gg D_{0}$ and a strong down-chirp $\left(\beta \simeq 3 \alpha_{l} / 2+\left(9 \alpha_{l}^{2} / 4+2\right)^{1 / 2}>0\right)$ for $D \ll 0$. Introducing the second term in the expansion of the exponent [Eq. (1)] for the gain, $\alpha_{l}$ in Eq. (6) should be replaced by $\left(\alpha_{l} l-\alpha_{g} g / s^{2}\right) /\left(l-g / s^{2}\right)$ for solution of the chirp parameter. This is in agreement with previous results. ${ }^{11,12}$ In the remainder of this paper, we neglect the contribution of $\alpha_{g}$ to $\beta$.

Since we require a steady train of pulses, the result is limited by the inequalities of net loss in the leading edge of the pulse

$$
l_{t}+l-g>0
$$

and after the passage of the pulse

$$
-\left(l_{t}+l-g\right)+\left(l-\frac{g}{s}\right) \frac{E}{E_{\mathrm{sl}}}-\frac{1}{2} l\left(\frac{E}{E_{\mathrm{sl}}}\right)^{2}<0 .
$$

Also, we require a net gain with rising pulse energy,

$$
l-g / s>0 .
$$

We now turn to the calculation of optical spectra given by the Fourier series of $a(t)$. Assuming that a large number of longitudinal modes exist in the laser cavity (as required for the WDM source and controlled by the cavity length and the material index of refraction), the envelope of the optical spectra $a(\omega)$ is given approximately in terms of the Fourier transform of $a(t)$,

$$
\begin{aligned}
a(\omega) \simeq & \int_{-\infty}^{\infty} a(t) \exp (-i \omega t) \mathrm{d} t=A \frac{2^{i \beta} \tau}{\Gamma(1+i \beta)} \\
& \times \Gamma\left[\frac{1}{2}+\frac{i}{2}(\beta+\omega \tau)\right] \Gamma\left[\frac{1}{2}+\frac{i}{2}(\beta-\omega \tau)\right],
\end{aligned}
$$

where $\Gamma$ ( ) is the gamma function, ${ }^{13}$ and it is understood that frequency $\omega$ is evaluated with respect to the carrier frequency. Also,

$$
|a(\omega)|^{2}=\frac{2 A^{2} \tau^{2} \pi^{3} \beta}{\sinh (\pi \beta)[\cosh (\pi \omega \tau)+\cosh (\pi \beta)]},
$$

with frequency FWHM $(\Delta f)$ given by

$$
\cosh \left[\Delta f \Delta \tau \frac{\pi^{2}}{2 \cosh ^{-1}(\sqrt{2})}\right]=\cosh (\pi \beta)+2,
$$

where $\Delta \tau=2 \tau \cosh ^{-1}(\sqrt{2})$ is the FWHM of $|a(t)|^{2}$. Therefore the magnitude of the chirp parameter can be measured directly from the time-bandwidth product $\Delta f \Delta \tau$. In the case of zero chirp $(\beta=0)$, the familiar result of $\Delta f \Delta \tau=0.3148$ is obtained. Equations (18)-(20) are general for pulse shapes in
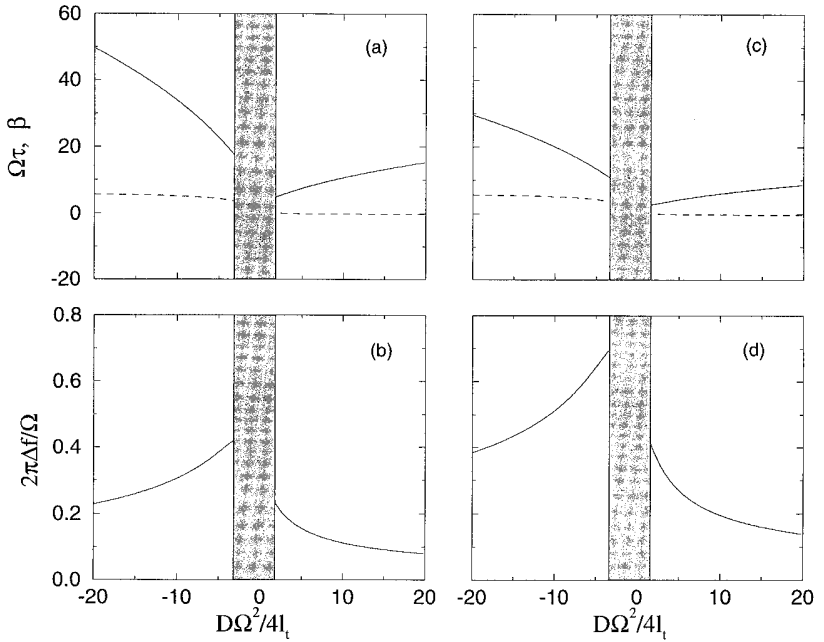

Fig. 1. Pulse width $\tau$, chirp parameter $\beta$ (dashed curve), and FWHM of the optical spectra profile $\Delta f$ as a function of normalized GVD. (a), (b), $l / l_{t}=1.4, g / l_{t}=2.13, s=8, \alpha_{g}=4$, and $\alpha_{l}=2$; (c), $(d), l / l_{t}=3.16, g / l_{t}=3.38, s=12, \alpha_{g}=6$, and $\alpha_{l}=2$. The gray areas represent unstable regions for which no stable solution was found.

the form given in Eq. (5) and are not restricted to mode-locked semiconductor lasers. The spectral phase is given by $\arg [a(\omega)]$, the argument of $a(\omega)$.

In Fig. 1 the pulse width, chirp parameter, and FWHM of the optical spectra are plotted as a function of the normalized GVD. For Figs. 1(a) and 1(b), the laser parameters are $l=1.4 l_{t}, g=2.13 l_{t}, s=8, \alpha_{l}=$ 2 , and $\alpha_{g}=4$, whereas in Figs. 1(c) and 1(d), the parameters are $l=3.16 l_{t}, g=3.38 l_{t}, s=12, \alpha_{l}=2$, and $\alpha_{g}=6$. The increase in $\Delta f$ in Fig. 1(d) compared with Fig. 1(b) is a result of pulse shortening because the chirp parameter is equal in both cases.

Although GVD changes the temporal profile of an optical signal through a change in the frequencydependent phase (frequency chirp), it does not directly change the spectral profile. The dependence of $\Delta f$ on $D$ in Fig. 1 results from a change in the action of saturable gain and loss through a change in GVD. A change in GVD affects the pulse shape by changing the dependence of pulse energy on time, i.e., the pulse energy spreads on a different time scale. This has an effect on the nonlinear terms, which in turn affect the optical spectrum profile. An important result stems from Fig. 1. The spectral width is wider by 60-80\% for negative (nonnormal) GVD. The gray area represents an unstable region resulting from inequality (16). For a higher pulse energy $\left(E \gg E_{\mathrm{sl}}\right)$ the unstable region is expected to narrow considerably or disappear.

It should be understood that, although the action of GVD is to produce linear frequency chirp by effectively redistributing in time the various frequency components of an optical signal, the action of SPM is to produce nonlinear frequency chirp by shifting some of the frequency components to new frequencies. The action of SPM on the spectral width is illustrated in Fig. 2 for various values of 


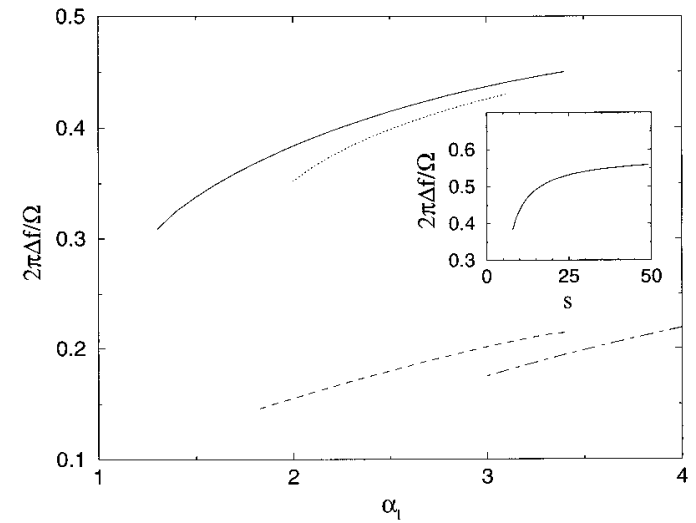

Fig. 2. Normalized FWHM of the optical spectra as a function of saturable absorber SPM parameter $\left(\alpha_{l}\right)$ for various values of saturable gain self-phase modulation parameter $\left(\alpha_{g}\right)$ and GVD. The laser parameters are $l=1.4 l_{t}, g=2.13 l_{t}$, and $s=8$. The solid curve represents the result for $\alpha_{g}=4$ and $D \Omega^{2} / 4 l_{t}=-5$; the dot-dash curve, $\alpha_{g}=6$ and $D \Omega^{2} / 4 l_{t}=-5$; the dashed curve, $\alpha_{g}=$ 4 and $D \Omega^{2} / 4 l_{t}=5$; the dot-dash curve represents $\alpha_{g}=6$ and $D \Omega^{2} / 4 l_{t}=5$. The inset illustrates the dependence of $\Delta f$ on the stability parameter $s$ for $\alpha_{g}=4, \alpha_{l}=2$, and $D \Omega^{2} / 4 l_{t}=-5$.

gain SPM, GVD, and the stability parameter. A strong, almost linear dependence was found on $\alpha_{l}$ (an approximately 6.3\% increase in the spectral width-per-unit increase in absorber SPM for the laser parameters represented by the solid curve in Fig. 2 and $\alpha_{l} \simeq 2$ ). This can be simply understood because photons at new frequencies are created by the saturable absorber, and so the spectral width of the optical signal is increased as SPM increases. Although the stability parameter is much larger than 1 , a weaker dependence was found on $\alpha_{g}$. Note that an increase of $\alpha_{g}$ decreases the spectral profile because a change in $\alpha_{g}$ has essentially no effect on the pulse chirp but does affect the pulse energy and its width through the action of GVD and therefore changes the spectral profile. As indicated by the inset in Fig. 2, a change in stability parameter $s$ also has a strong effect on the spectral width through a change in self-amplitude modulation of the gain. For higher values of $s$ this dependence is weakened.

An important result is that the nonlinear coupling between longitudinal modes, caused by the action of self-amplitude and phase modulation, can result in broad optical spectra in which a large fraction of the lasing longitudinal modes obtain equal power. As illustrated by the solid curve in Fig. 3(a), this happens when a large absolute value of the chirp parameter is achieved, i.e., for negative (nonnormal) GVD. This result can be utilized in WDM sources for which a broad spectrum is required although the value of the chirp parameter is unimportant because each mode serves as an individual beam that is modulated and carries information independently from other modes. In comparison, the dashed curve in Fig. 3(a) represents the same laser parameters but with positive (normal) GVD. Because the achieved chirp is
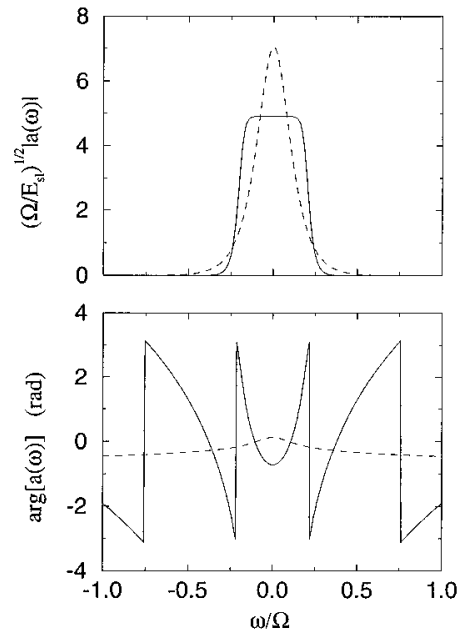

Fig. 3. (a) Normalized spectral amplitude $\left(\Omega / E_{\mathrm{sl}}\right)^{1 / 2}|\mathrm{a}(\omega)|$ and (b) spectral phase $\arg [a(\omega)]$ versus the normalized angular frequency. The laser parameters are $l / l_{t}=1.4, g / l_{t}=2.13$, and $s=8$. The solid curves $(\beta=4.32)$ illustrate the negative normalized GVD $\left(D \Omega^{2} / 4 l_{t}=-5\right)$, whereas the dashed curves represent $D \Omega^{2} / 4 l_{t}=$ $5(\beta=-0.179)$.

much smaller in absolute value, this results in a narrower sech-like spectral profile.

Figure 3(b) illustrates the modal phase, i.e., the dependence of spectral phases on frequency. As the solid curve indicates, a strong frequency down-chirp of the optical-pulse electric field for negative GVD is found. Again, in comparison, for positive (normal) GVD we achieved a small up-chirp (dashed curve).

In conclusion, the possibility of utilizing a passively mode-locked semiconductor laser as a WDM source was theoretically considered. For high values (limited by stability requirements) of SPM of the absorption section $\left(\alpha_{l}\right)$ and intermediate values of negative (nonnormal) GVD [slightly above the minimal value needed for stabilization (see Fig. 1)] a large downchirp can be achieved resulting in a broad squarelike optical spectra profile. A control over the chirp has been achieved before. ${ }^{11}$ A strong down-chirp was observed for high gain bias and a higher ratio of absorber-to-gain section lengths. For a laser operating at higher power for which the approximation of low energy is no longer valid, we expect to use this model to describe qualitatively the optical spectrum and its dependence on laser parameters. Also, higher power can result in asymmetry of pulse shape and optical spectrum because higher terms in the Taylor expansion of the exponents in Eq. (1) are important.

Finally, we note that these advantages of the nonlinearity (broad optical spectra), together with those of additional stability of active mode locking, can be utilized in so-called hybrid mode-locking lasers. ${ }^{5}$

The authors are pleased to acknowledge Roger G. M. P. Koumans for useful discussions. This research was supported by the Advanced Research Projects Agency. 


\section{References}

1. T. Morioka, K. Mori, and M. Saruwatari, "More than 100wavelength-channel picosecond optical pulse generation from single-laser source using supercontinuum in optical fibers," Electron. Lett. 29, 862-864 (1993).

2. H. W. Messenger, "Developers unveil modelocked erbium fiber laser," Laser Focus World 32, 15-16 (May 1996).

3. B. Y. Zhu and I. H. White, "Multiwavelength picosecond optical pulse generation using an actively mode-locked multichannel grating cavity laser,” J. Lightwave Technol. 13, 2327-2335 (1995).

4. H. Hasaka, Y. Yoshikuni, K. Sato, H. Ishii, and H. Sanjoh, "Multiwavelength light source with precise frequency spacing using mode-locked semiconductor laser and arrayed waveguide grating filter," in Optical Fiber Communication Conference, Vol. 2 of 1996 OSA Technical Digest Series (Optical Society of America, Washington, D.C., 1996), pp. 299-300.

5. D. J. Derickson, R. J. Helkey, A. Mar, J. R. Karin, J. G. Wasserbauer, and J. E. Bowers, "Short pulse generation using multisegment mode-locked semiconductor-lasers," IEEE J. Quantum Electron. 28, 2186-2202 (1992).

6. H. A. Haus, "Theory of mode-locking with slow saturable absorber," IEEE J. Quantum Electron. QE-11, 736-746 (1975).

7. O. E. Martinez, R. L. Fork, and J. P. Gordon, "Theory of passively mode-locked lasers for the case of a nonlinear com- plex propagation coefficient,” J. Opt. Soc. Am. B 2, 753-760 (1985).

8. R. G. M. P. Koumans, and R. van Roijen, "Theory for passive mode-locking in semiconductor laser structures including the effects of self-phase modulation, dispersion, and pulse collisions," IEEE J. Quantum Electron. 32, 478-492 (1996); R. G. M. P. Koumans, R. A. Salvatore, D. Eliyahu, and R. van Roijen, "Correction to: Theory for passive mode-locking in semiconductor laser structures including the effects of selfphase modulation, dispersion, and pulse collisions," IEEE J. Quantum Electron. 32, 2017 (1996).

9. G. H. C. New, "Pulse evolution in mode-locked quasicontinuous lasers," IEEE J. Quantum Electron. QE-10, 115124 (1974).

10. E. P. Ippen, "Principles of passive mode locking," Appl. Phys. B 58, 159-170 (1994).

11. R. A. Salvatore and A. Yariv, "Demonstration of down-chirped and chirp-free pulses from high-repetition-rate passively mode-locked lasers," IEEE Photon. Technol. Lett. 7, 11511153 (1995).

12. M. Schell, J. Yu, M. Tsuchiya, and T. Kamiya, "Chirp of passively and actively mode-locked semiconductor-lasers," Appl. Phys. Lett. 67, 1797-1799 (1995).

13. I. S. Gradshteyn and I. M. Ryzhik, Table of Integrals, Series and Products, 4th ed. (Academic, New York, 1965), p. 933. 